\title{
The Efficacy of an Octenidine Decolonisation Protocol in Reducing Staphylococcus Aureus Carriage in Patients Undergoing Total Joint Arthroplasty
}

\author{
Tristan Symonds ${ }^{1, *}$, Hannah Brien ${ }^{2}$, Benjamin Parkinson ${ }^{1,2}$, Andrea Grant ${ }^{1}$, Kenji Doma $^{1}$ \\ ${ }^{1}$ Orthopaedic Research Institute of Queensland, Townsville, Queensland \\ ${ }^{2}$ Cairns Private Hospital, Cairns, Queensland
}

\section{Email address:}

Tristan.symonds@my.jcu.edu.au (T. Symonds), BrienH@ramsayhealth.com.au (H. Brien), Benjaminparkinson@gmail.com (B. Parkinson), research_coordinator@oriql.com.au (A. Grant), Kenji.doma@jcu.edu.au (K. Doma)

${ }^{*}$ Corresponding author

\section{To cite this article:}

Tristan Symonds, Hannah Brien, Benjamin Parkinson, Andrea Grant, Kenji Doma. The Efficacy of an Octenidine Decolonisation Protocol in Reducing Staphylococcus Aureus Carriage in Patients Undergoing Total Joint Arthroplasty. Journal of Surgery. Vol. 8, No. 3, 2020 , pp. 81-85. doi: $10.11648 /$ j.js.20200803.11

Received: April 7, 2020; Accepted: April 26, 2020; Published: May 19, 2020

\begin{abstract}
Prosthetic joint infections are responsible for a high morbidity and economic cost in patients undergoing total joint arthroplasty. Staphylococcus aureus (S Aureus) is a key modifiable risk factor in the reduction of surgical site infections (SSI). Evidence suggests that decolonization of $S$ aureus carriers reduces the risk of SSI. Octenidine is an antiseptic active against gram-positive and gram-negative bacteria. The aim of this study is to establish the eradication rate of $S$ aureus in patients undergoing total joint arthroplasty using an Octenidine decolonisation protocol prior to surgery. This was a prospective case series performed at the Cairns Private Hospital during a five month trial period of using Octenidine wash and nasal gel in patients undergoing elective TJA. Patients undergoing total joint arthroplasty during a five-month trial period had pre-operative nasal swabs to screen for S aureus pre and post Octenidine treatment. All patients underwent a body wash and nasal gel protocol for five days using Octenidine. The primary outcome was to determine decolonisation rates in patients following the protocol. A total of 183 patients met inclusion criteria into this study. At the first swab $151(82.5 \%)$ patients had normal regional flora and 32 $(17.5 \%)$ were positive for $\mathrm{S}$ aureus. Of these 32 patients $30(93.75 \%)$ were negative for MRSA and $2(6.25 \%)$ had non-multiresistant MRSA. The decolonization rate for patients with S aureus was $76.6 \%$. It was unsuccessful in clearing the two patients with nmMRSA. Octenidine is effective in reducing $S$ aureus colonisation in patients undergoing total joint arthroplasty. Further studies are required to compare this agent to traditional mupirocin based protocols to determine its efficacy as an alternative for use in pre-operative staphylococcal decolonisation.
\end{abstract}

Keywords: Total Joint Arthroplasty, Decolonization, Octenidine, Staphylococcus Aureus, Prosthetic Joint Infections

\section{Introduction}

Prosthetic joint infection is a devastating complication for patients to suffer and it carries considerable social and economic cost. Modifiable risk factors are increasingly being identified to optimise patient outcomes. Staphylococcus aureus colonisation has been recognised as a key modifiable risk factor for surgical site infections (SSI) in patients who are undergoing elective total joint arthroplasty (TJA). [1]

Patients undergoing TJA are typically treated under a "screen and treat" protocol. This protocol involves patients being screened at a pre-admission clinic for $S$ aureus and if tested positive undergoing a decolonisation protocol prior to surgery. Several studies have been conducted on nasal decolonisation of patients undergoing orthopedic surgery by using perioperative intranasal mupirocin. [2-4] Intranasal mupirocin and chlorhexidine wash is the gold standard antimicrobial for decolonization of $S$ aureus and has been shown to eradication rates between $81.5-100 \%$ following a course of treatment. [5,6] This is an attractive prevention strategy being a safe and simple method to eradicate nasal colonization. The most common protocol for this is $2 \%$ 
intranasal mupirocin and chlorhexidine gluconate for three to five days prior to surgery. This is sufficient to show a reduction both in colonisation rates and in $S$ aureus SSI. [1]

A potential issue is the emergence of mupirocin-resistant $S$ aureus, which in many cases is on the rise and is associated with unsuccessful decolonisation prior to surgery. [7, 8] A rise in mupirocin resistance will lead to a reduced effectiveness in mupirocin decolonisation protocols and increased SSIs.

An alternative to the "screen and treat" protocol is a universal decolonisation protocol where all patients undergoing elective TJA undergo a decolonisation protocol without screening. A recent study using a combination of mupirocin with chlorhexidine in a universal decolonisation protocol was shown to reduce the overall rate of SSIs. [9] The concerns with mupirocin resistance are obviously further amplified with these universal decolonisation protocols and this has led to the investigation of alternative antiseptic agents such as nasal povidone-iodine and octenidine.

Octenidine Dihydrochloride (Octenidine) is a modern antiseptic for skin, mucous membranes and wounds introduced more than 25 years ago. It is established as an antiseptic in a large field of applications and represents an alternative to the older substances such as chlorhexidine, iodine or triclosan. Octenidine is a cationic surfactant, active against gram-positive and gram-negative bacteria. Octenidine is neither absorbed through the skin, nor through mucous membranes, nor via wounds.[10,11] These properties make Octenidine a potential alternative for a decolonization protocol, as at appropriate concentrations will not allow bacteria to develop resistance. [12]

This study aims to investigate the decolonisation rates of Octenidine in patients undergoing elective TJA to determine if it would be comparable to traditional decolonisation protocols. There is currently no existing published data on the use of Octenidine in arthroplasty patients.

\section{Method}

The study obtained ethics approval following review by the hospital research and ethics committee. The study was a prospective interventional pilot study performed at the Cairns Private Hospital during a five month trial period of using Octenidine wash and nasal gel in patients undergoing elective TJA. Patients undergoing TJA had a pre-treatment nasal swab in the pre-admission clinic. All patients were required to use Octenidine body wash and nasal gel twice a day for five days prior to surgery. Patients then had a repeat nasal swab performed on the day of surgery.

Inclusion criteria for the study were patients who were undergoing TJA at the Cairns Private Hospital during their five month trial of Octenidine decolonisation. Exclusion criteria were patients who were non-compliant with the protocol and those who did not have both nasal swabs performed.

The primary outcome was to determine the decolonization rates with Octenidine body wash and nasal gel. Patients who had a swab which grew $S$ aureus were identified as being colonized. The decolonization rates were determined by patients who were colonized by $S$ aureus at preadmission clinic and were negative for $S$ aureus at time of surgery after using the wash.

An additional outcome was to determine the patient population that were non-compliant with the wash protocol. This was done via patient self-reported compliance with the wash at the time of surgery. These patients were excluded from data used to identify decolonization rates; however, provide important information as to compliancy rates amongst patients if a universal decolonization protocol was to be implemented.

Microbiology

$\mathrm{ESwab}^{\circledR}$ sterile transport swabs were used for collection from patients. Swabs were transported in liquid Amies solution to the laboratory. Swabs were set on horse blood agar and chromogenic MRSA plates. Plates were incubated in a $\mathrm{CO} 2$ Incubator. Total incubation time was 48 hours with plates examined for Staph growth at 24 and 48 hours.

\section{Results}

Figure 1 is a Consort flow diagram giving visual representation to the following.

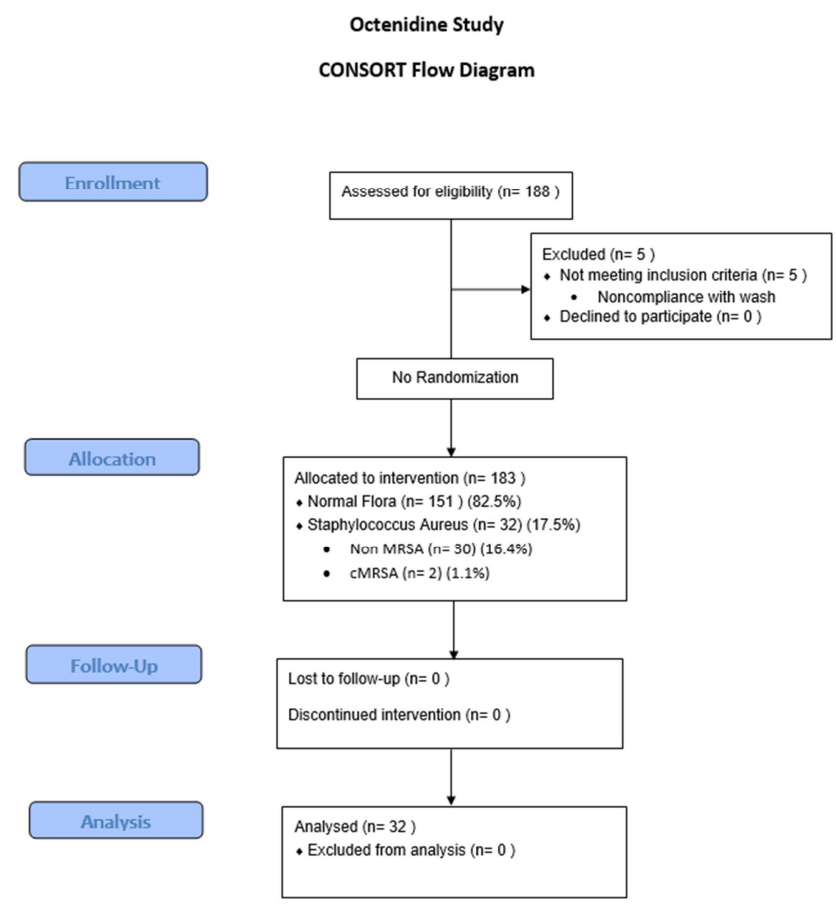

Figure 1. Octenidine Study Consort Flow Diagram.

There were 188 patients who met the criteria for inclusion into the study. A total of 5 patients were excluded for non-compliance with the wash protocol, this left 183 patients who met inclusion criteria. At preadmission clinic 151 (82.5\%) patients had normal regional flora isolated on their swab and $32(17.5 \%)$ patients were positive for $S$ aureus. Of these patients, 30 were positive for $S$ aureus (Negative for MRSA) and 2 were positive for non-multiresistant MRSA (nmMRSA)

Table 1 outlines the results for patients who were colonized 
with $S$ aureus at the preadmission clinic and the decolonization rate based on the result of their subsequent swab on the day of surgery. Patients who were positive for $S$ Aureus had a decolonization rate of $76.6 \%$ following the wash protocol. The two patients who were positive for nmMRSA were unsuccessful with decolonization following the wash protocol.

Table 1. Staphylococcus Aureus Colonization and Decolonization rate.

\begin{tabular}{llll}
\hline & Preadmission Swab & Swab Day of Surgery & Decolonization Rate \\
\hline S Aureus (Negative MRSA) & 30 & 7 & $76.6 \%$ \\
S Aureus (non-multiresistant MRSA) & 2 & 2 & $0 \%$ \\
Total & 32 & 9 & $71.8 \%$ \\
\hline
\end{tabular}

Table 2 outlines the clearance rate with octenadine obtained in the current study, in comparison to the clearance rates with mupirocin and placebo based on previous studies. According to the Goodness of Fit Chi-squared test, no significant differences were found in clearance rate between octenadine and mupirocin $\left(p=0.051 ; \chi^{2}=3.79\right)$, whilst significant differences were identified between octenadine and placebo $(\mathrm{p}$ $\left.<0.001 ; \chi^{2}=30.3\right)$.

Table 2. Decolonization rate with octenadine, mupirocin and placebo.

\begin{tabular}{llll}
\hline & Cleared & Not cleared & Decolonisation rate \\
\hline Octenadine & 23 & 9 & $71.8 \%$ \\
Mupirocin & 27 & 5 & $85.3 \% *$ \\
Placebo & 9 & 23 & $26.7 \% *$ \\
\hline
\end{tabular}

*Calculated using previous data [13-15]

\section{Discussion}

This study has shown that an Octenidine decolonisation protocol prior to surgery is successful in eradicating $S$ aureus carriage in elective total joint arthroplasty patients in $71.8 \%$ of cases. This is just below the published decolonisation rate for chlorhexidine and mupirocin protocols. [5]

There is currently no existing published data on the use of Octenidine as a decolonisation agent in arthroplasty patients. The published literature on octenidine decolonisation is mostly limited to observational studies on MRSA colonised patients in ICU settings. A review of the studies on MRSA colonised patients by Krishna and Gibb found decolonisation rates for octenidine were reported between $6-75 \%$. [16] The major limitation of the existing studies is they examined patients colonized with MRSA and had varied decolonisation protocols ranging from Octenidine alone to a combination with mupirocin. [17, 18] The studies were all observational with total number of subjects ranging from 5-126. [19-21] This data is of limited use in our elective arthroplasty population where MRSA colonisation is not a frequent occurrence.

Decolonisation rates of mupirocin have been established to be between $81.5-100 \%$. [5] This rate is likely to be lower under everyday working conditions were compliance may reduce the effect further. [20]The secondary outcome from our study showed that $2.6 \%$ of patients were noncompliant with the wash protocol. Compliance rates in studies examining mupirocin decolonisation protocols vary from often unreported, to between $31.1->90 \%$. [22, 23] It is hypothesized that the compliance rate will be higher in a universal decolonisation protocol vs screen and treat, although there is no evidence available.

A recent study by Stambough JB et al. compared a universal decolonisation protocol versus a "screen and treat" protocol.
[9] The study showed the universal decolonisation protocol resulted in a significant decrease in both overall SSI and SSI related to $S$ aureus organisms. Additionally, the universal decolonisation protocol demonstrated a significant potential for economic gains for the health system as a result of limiting future reoperations and hospitalisations. This study did not report the clearance rates of the $S$ aureus between the universal and screen and treat groups.

Emerging evidence suggests that increased use of mupirocin has been associated with developing resistance through enhanced selective pressures and cross resistance. This has diminished the effectiveness of decolonisation protocols and led to treatment failure. [24] The emergence of resistance is being reported in many parts of the world at different frequencies: Spain 11.3\%, USA 13.2\%, Trinidad Tobago $26.1 \%$, China $6.6 \%$, India $6 \%$, Turkey $45 \%$, and Korea 5\%. [25] This has led to hesitation by many hospitals to universally give patients mupirocin despite published economic and health benefits. The major advantage of using an octenidine antiseptic protocol is that the issues with developing mupirocin antibiotic resistance can be avoided. [12]

The limitations of this study are that does not have a comparator group. We can therefore make no direct conclusions between the eradication efficacies of traditional mupirocin vs Octenidine decolonisation protocols. For this reason a Goodness of Fit Chi-squared test was conducted to make indirect comparisons of the results of our studies to previous studies. This suggested that there was no significant difference in decolonisation between Octenidine and Mupirocin ( $\mathrm{p}=0.051)$, whilst there were significant differences in decolonisation between Octenidine and placebo $(p<0.001)$. A limitation of the secondary outcome is the self-reported compliance. This may be verified in future studies if there is concurrent validity. Future studies comparing chlorhexidine / 
mupirocin protocols with octenidine protocols in similar patient populations are required.

The results from this study showed that a five-day decolonisation protocol with Octenidine provided a decolonisation rate of $71.8 \%$ in patients with $S$ aureus carriage. The two patients with nmMRSA were not successfully decolonised with this protocol but we would be hesitant to make any conclusions about nmMRSA until a larger sample size of these patients can be studied.

\section{Conclusion}

Octenidine is an attractive option for a universal decolonisation protocol in patients undergoing TJA as its mechanism of action means it is unlikely that bacteria will be able to build resistance. This study demonstrates that an Octenidine wash and nasal gel decolonisation protocol reduces $S$ aureus colonisation and that non-compliance rates are lower than previously reported.

Further studies are needed to directly compare its efficacy to traditional mupirocin protocols and also evaluate insurgence of possible cross resistance.

\section{References}

[1] Chen AF, Wessel CB, Rao N. Staphylococcus aureus screening and decolonization in orthopaedic surgery and reduction of surgical site infections. Clinical orthopaedics and related research. 2013; 471 (7): 2383-99.

[2] Wilcox MH, Hall J, Pike H, Templeton PA, Fawley WN, Parnell $\mathrm{P}$, et al. Use of perioperative mupirocin to prevent methicillin-resistant Staphylococcus aureus (MRSA) orthopaedic surgical site infections. The Journal of hospital infection. 2003; 54 (3): 196-201.

[3] Gernaat-van der Sluis AJ, Hoogenboom-Verdegaal AM, Edixhoven PJ, Spies-van Rooijen NH. Prophylactic mupirocin could reduce orthopedic wound infections. 1,044 patients treated with mupirocin compared with 1,260 historical controls. Acta orthopaedica Scandinavica. 1998; 69 (4): 412-4.

[4] Rao N, Cannella B, Crossett LS, Yates AJ, Jr., McGough R, 3rd A preoperative decolonization protocol for staphylococcus aureus prevents orthopaedic infections. Clinical orthopaedics and related research. 2008; 466 (6): 1343-8.

[5] Coates T, Bax R, Coates A. Nasal decolonization of Staphylococcus aureus with mupirocin: strengths, weaknesses and future prospects. The Journal of antimicrobial chemotherapy. 2009; 64 (1): 9-15.

[6] Tsang STJ, McHugh MP, Guerendiain D, Gwynne P, Boyd J, Laurenson IF, et al. Evaluation of Staphylococcus aureus eradication therapy in orthopaedic surgery. Journal of Medical Microbiology. 2018; 67 (6): 893-901.

[7] Fujimura S, Watanabe A. Survey of high- and low-level mupirocin-resistant strains of methicillin-resistant Staphylococcus aureus in 15 Japanese hospitals. Chemotherapy. 2003; 49 (1-2): 36-8.

[8] Simor AE, Stuart TL, Louie L, Watt C, Ofner-Agostini M, Gravel D, et al. Mupirocin-resistant, methicillin-resistant
Staphylococcus aureus strains in Canadian hospitals. Antimicrobial agents and chemotherapy. 2007; 51 (11): 3880-6.

[9] Stambough JB, Nam D, Warren DK, Keeney JA, Clohisy JC, Barrack RL, et al. Decreased Hospital Costs and Surgical Site Infection Incidence With a Universal Decolonization Protocol in Primary Total Joint Arthroplasty. The Journal of arthroplasty. 2017; 32 (3): 728-34. e1.

[10] Axel Kramer OA. Octenidine, Chlorhexidine, Iodine and Iodophores. Preprint from Wallhäußers Praxis der Sterilisation, Antiseptik und Konservierung: Thieme Georg Verlag; 2008.

[11] Assadian O. Octenidine dihydrochloride: chemical characteristics and antimicrobial properties. Journal of wound care. 2016; 25 (3 Suppl): S3-6.

[12] Al-Doori Z, Goroncy-Bermes P, Gemmell CG, Morrison D. Low-level exposure of MRSA to octenidine dihydrochloride does not select for resistance. The Journal of antimicrobial chemotherapy. 2007; 59 (6): 1280-1.

[13] Perl TM, Cullen JJ, Wenzel RP, Zimmerman MB, Pfaller MA, Sheppard D, et al. Intranasal mupirocin to prevent postoperative Staphylococcus aureus infections. The New England journal of medicine. 2002; 346 (24): 1871-7.

[14] Doebbeling BN, Breneman DL, Neu HC, Aly R, Yangco BG, Holley HP, Jr., et al. Elimination of Staphylococcus aureus nasal carriage in health care workers: analysis of six clinical trials with calcium mupirocin ointment. The Mupirocin Collaborative Study Group. Clinical infectious diseases: an official publication of the Infectious Diseases Society of America. 1993; 17 (3): 466-74.

[15] Konvalinka A, Errett L, Fong IW. Impact of treating Staphylococcus aureus nasal carriers on wound infections in cardiac surgery. The Journal of hospital infection. 2006; 64 (2): 162-8.

[16] Krishna BV, Gibb AP. Use of octenidine dihydrochloride in meticillin-resistant Staphylococcus aureus decolonisation regimens: a literature review. The Journal of hospital infection. 2010; 74 (3): 199-203.

[17] Rohr U, Mueller C, Wilhelm M, Muhr G, Gatermann S. Methicillin-resistant Staphylococcus aureus whole-body decolonization among hospitalized patients with variable site colonization by using mupirocin in combination with octenidine dihydrochloride. The Journal of hospital infection. 2003; 54 (4): 305-9.

[18] Sloot N, Siebert J, Hoffler U. Eradication of MRSA from carriers by means of whole-body washing with an antiseptic in combination with mupirocin nasal ointment. Zentralblatt fur Hygiene und Umweltmedizin=International journal of hygiene and environmental medicine. 1999; 202 (6): 513-23.

[19] Rengelshausen J, Nürnberger J, Philipp T, Kribben A. Decolonization of methicillin-resistant $<$ em $>$ Staphylococcus aureus $</$ em $>$ by disinfection of the skin. The American Journal of Medicine. 2000; 108 (8): 685-6.

[20] Hansen D, Patzke PI, Werfel U, Benner D, Brauksiepe A, Popp W. Success of MRSA eradication in hospital routine: depends on compliance. Infection. 2007; 35 (4): 260-4.

[21] Pichler G PC, Babeluk R, et al. MRSA prevalence rates detected in a tertiary care hospital in Austria and successful treatment of MRSA positive patients applying a decontamination regime with octenidine. Eur J Clin Microbiol Infect Dis. 2017; 37 (21). 
[22] Fritz SA, Camins BC, Eisenstein KA, Fritz JM, Epplin EK, Burnham CA, et al. Effectiveness of measures to eradicate Staphylococcus aureus carriage in patients with community-associated skin and soft-tissue infections: a randomized trial. Infection control and hospital epidemiology. 2011; 32 (9): 872-80.

[23] Caffrey AR, Woodmansee SB, Crandall N, Tibert C, Fielding C, Mikolich DJ, et al. Low adherence to outpatient preoperative methicillin-resistant Staphylococcus aureus decolonization therapy. Infection control and hospital epidemiology. 2011; 32 (9): 930-2.
[24] Hetem DJ, Bonten MJ. Clinical relevance of mupirocin resistance in Staphylococcus aureus. The Journal of hospital infection. 2013; 85 (4): 249-56.

[25] Rajkumari N, Mathur P, Bhardwaj N, Gupta G, Dahiya R, Behera B, et al. Resistance pattern of mupirocin in methicillin-resistant Staphylococcus aureus in trauma patients and comparison between disc diffusion and E-test for better detection of resistance in low resource countries. J Lab Physicians. 2014; 6 (2): 91-5. 